\title{
A CLASS OF SINGULARLY PERTURBED EVOLUTION SYSTEMS
}

\author{
N.U. AHMED \\ University of Ottawa \\ Department of Mathematics and \\ Department of Electrical Engineering \\ 161 Louis Pasteur \\ Ottawa, Ontario \\ CANADA K1N $6 N 5^{1}$
}

(Received November, 1993; revised May, 1994)

\begin{abstract}
In this paper we study a class of evolution equations where the semigroup generators are singularly perturbed by a nonnegative real valued function of time. Sufficient conditions for existence of evolution operators and their compactness are given including continuous dependence on the perturbation. Further, for a coupled system of singularly perturbed semilinear systems in two Banach spaces, existence of periodic solutions and their stability are studied
\end{abstract}

Key words: Semigroups, Singular Perturbation, Evolution Equations, Nonexpansive Maps, Existence, Periodic Solutions.

AMS (MOS) subject classifications: $34 \mathrm{~K} 30,35 \mathrm{~A} 05,34 \mathrm{D} 15,34 \mathrm{C} 25,47 \mathrm{D} 03$, $47 \mathrm{H} 20$.

\section{Introduction}

First, we introduce some notations. For any pair of Banach spaces $X$ and $Y, \ell(X, Y)$ will denote the class of bounded linear operators from $X$ to $Y$. For $\ell(X, X)$ we use the notation $\mathcal{L}(X)$ for short. For $1 \leq p \leq \infty, L_{p}^{\text {loc }}([0, \infty), \mathcal{L}(X, Y))$ will denote the class of uniformly measurable $\mathcal{L}(X, Y)$-valued functions whose norms are locally $p$-th power integrable in the Lebesgue sense. For any Banach space $X$ and $M \geq 1$ and $\omega \in \mathbb{R}$, we let $G(X, M, \omega)$ denote the class of infinitesimal generators of $C_{0}$-semigroups $\{T \equiv\{T(t), t \geq 0\}\}$, with stability parameters $\{M, \omega\}$, that is $\|T(t)\|_{\mathcal{L}(X)} \leq M e^{\omega t}, t \geq 0$.

Before proceeding with the general problem we present some examples that inspired us to consider systems of the form

$$
\begin{gathered}
(d / d t) x=\beta(t) A x+f(x), \quad t \geq 0, \\
x(0)=x_{0} .
\end{gathered}
$$

Though the linear part appears to be a special class of general nonautonomous operators $A(t)$

${ }^{1}$ This work was supported in part by the National Science and Engineering Research Council of Canada under grant No. A7109.

Printed in the U.S.A. C)1994 by North Atlantic Science Publishing Company 
which have been studied extensively in the literature (see Ladas, Lakshmikantham [6]), it has many interesting special properties and applications. Further, the assumptions required for the general case are far more stronger than those imposed here on $\beta(t), t \geq 0$, or equivalently $A(t) \equiv \beta(t) A$. In the general case, typical assumptions are: for each $t, A(t)$ is the generator of an analytic semigroup, $0 \in \rho(A(t)), t \rightarrow A(t) A^{-1}(0)$ is Hölder continuous, etc. For our special class, none of these are necessary.

\section{Some Examples:}

(E1): Consider the stochastic differential equation in $\mathbb{R}^{n}$ :

$$
d \xi=b(\xi) d t+\sigma(\xi) d W, \quad t \geq 0
$$

where $W$ is an $\mathbb{R}^{d}$-valued standard Brownian motion on a filtered probability space $\left\{\Omega, \mathscr{F} \supset \mathscr{F}_{t} \uparrow P\right\}$. Under suitable assumptions on the coefficients $b, \sigma$ it is well known that $\xi=\{\xi(t), t \geq 0\}$ is a Markov diffusion process in $\mathbb{R}^{n}$ with infinitesimal generator $A$ given by

$$
A \psi \equiv(b, \nabla \psi)+(1 / 2) \operatorname{tr}\left(a \nabla^{2} \psi\right) ; a \equiv \sigma \sigma^{\prime}
$$

Now, for $\epsilon>\mathbb{0}$, consider the singularly perturbed equation

$$
\epsilon d \xi_{\epsilon}=b\left(\xi_{\epsilon}\right) d t+\sqrt{\epsilon} \sigma\left(\xi_{\epsilon}\right) d W, \quad t \geq 0 .
$$

By Ito's formula, (see Friedman [3]), it is easy to verify that the generator of the process $\xi_{\epsilon}$ is given by $A_{\epsilon} \equiv(1 / \epsilon) A$. Both $A$ and $A_{\epsilon}$ are the infinitesimal generators of Markov (contraction) semigroups on $X \equiv C_{b}\left(\mathbb{R}^{n}\right)$. Letting $T$ denote the semigroup corresponding to the generator $A$, it is clear that the semigroup $T_{\epsilon}$ corresponding to $A_{\epsilon}$ is given by $T_{\epsilon}(t)=T(t / \epsilon)$. Thus, for any $\phi_{0} \in X$,

$$
E\left\{\phi_{0}\left(\xi_{\epsilon}(t)\right) \mid \xi_{\epsilon}(0)=x\right\}=T_{\epsilon}(t) \phi_{0} \equiv T(t / \epsilon) \phi_{0} .
$$

Clearly, $T_{\epsilon}(t) \phi_{0}, t \geq 0$, is the solution of the singularly perturbed differential equation

$$
(d / d t) \phi=(1 / \epsilon) A \phi, \phi(0)=\phi_{0},
$$

in the Banach space $X \equiv C_{b}\left(\mathbb{R}^{n}\right)$. It is clear from this example that the effect of $\epsilon$ is only a change of time scale speeding up or slowing down the process. Similarly, for the equation

$$
d \xi_{\epsilon}=b\left(\xi_{\epsilon}\right) d t+\sqrt{\epsilon} \sigma\left(\xi_{\epsilon}\right) d W, \quad t \geq 0,
$$

the Kolmogorov equation is given by

$$
(d / d t) \phi=\epsilon A^{0} \phi+B \phi, \quad t \geq 0,
$$

where $A^{0} \psi \equiv(1 / 2) \operatorname{tr}\left(\sigma \sigma^{*} \psi_{x x}\right)$ and $B \psi \equiv\left(b, \psi_{x}\right)$. Here $A^{0}$ is the semigroup generator under suitable boundary conditions, such as homogeneous Dirichlet boundary condition in case of a stopped Markov process, and $B$ can be considered as a relatively bounded perturbation. For deeper study of such perturbed stochastic differential equations, see the excellent monograph of Friedman [3, chapter 14].

(E2): For the heat equation with time varying conductivity, $\beta(t) \geq 0$, we have

$$
(\partial / \partial t) \phi(t, \xi)-\beta(t) \Delta \phi(t, \xi)=f(t, \xi), t \geq 0, \xi \in \Sigma
$$




$$
\begin{gathered}
\phi(t, \xi)=0, \quad t \geq 0, \xi \in \partial \Sigma \\
\phi(0, \xi)=\phi_{0}(\xi), \quad \xi \in \Sigma
\end{gathered}
$$

where $\Sigma$ is a bounded open set in $\mathbb{R}^{3}$ with sufficiently smooth boundary $\partial \Sigma$. This situation may arise when different materials of varying conductivity are batched through the same furnace or boiler. This may also arise from change of the material properties and hence conductivity due to exposure to reacting gases. It may also arise from the approximation of a nonlinear heat equation replacing temperature dependent conductivity by it's spatial average.

Define the operator $A$ by

$$
\begin{gathered}
D(A) \equiv\left\{\phi \in L_{2}(\Sigma): \Delta \phi \in L_{2}(\Sigma),\left.\phi\right|_{\partial \Sigma}=0\right\} \\
A \phi=\Delta \phi, \text { for } \phi \in D(A) .
\end{gathered}
$$

In fact $D(A)=H_{0}^{1} \cap H^{2}$. It is known that $A$ generates a $C_{0}$-semigroup in $X \equiv L_{2}(\Sigma)$. Clearly equation (1.5) can be written as an abstract differential equation

$$
\begin{gathered}
(d / d t) u=\beta(t) A u+g(t), \quad t \geq 0 \\
u(0)=\phi_{0}
\end{gathered}
$$

in the Hilbert space $X=L_{2}(\Sigma)$ where $g(t) \equiv f(t, \cdot)$ is some $X$-valued function.

(E3): By standard techniques (see Goldstein [5], Ahmed [1]), the Navier Stokes equation for incompressible fluid can be formulated as an evolution equation:

$$
(d / d t) v+\gamma(t) A v=f(v), \quad t \geq 0
$$

in the Hilbert space $H$ obtained by the strong closure (in the topology of $L_{2}\left(\Sigma, \mathbb{R}^{n}\right)$ ) of divergence free $C_{0}^{\infty}\left(\Sigma, \mathbb{R}^{n}\right)$-vector fields. Here $v$ is the fluid velocity, $A$ is the Stokes operator which is positive and self adjoint, $\gamma$, known as the kinematic viscosity, is directly proportional to material viscosity and inversely proportional to Reynolds number. The material viscosity changes with time either due to temperature variation or due to material degradation with time. The nonlinear part arises from the convective term.

The paper is organized as follows. In Section 1, notations and some motivating examples are given. In Section 2, for linear and semilinear problems, sufficient conditions for existence of evolution operators and their compactness are presented including a result on continuous dependence of solutions on the perturbation. In Section 3, similar results are given for a pair of evolution equations. Further, some results on existence of periodic solutions and their stability are also given.

\section{Existence and Regularity of Solutions}

First we consider a single linear evolution equation of the form

$$
\begin{gathered}
(d / d t) x=\beta(t) A x+C(t) x, t \geq 0, \\
x(0)=x_{0} .
\end{gathered}
$$


We shall prove the following result.

Theorem 2.1: Let $\beta(t) \geq 0$ for all $t \geq 0, \beta \in L_{1}^{\text {loc }}(0, \infty), A \in G(X, M, \omega)$ and $T$ the corresponding semigroup of bounded linear operators in $X$. Then

(i) for $C \in L_{1}^{\text {loc }}([0, \infty), \mathcal{L}(X))$, the operator $\mathcal{A}(t) \equiv \beta(t) A+C(t), t \geq 0$, is the gener ator of a strongly continuous evolution operator $U(t, \theta), 0 \leq \theta \leq t<\infty$ in $X$;

(ii) for $C \in L_{\infty}^{\text {loc }}([0, \infty), \mathcal{L}(X))$, if $T$ is a compact semigroup and $\bar{\beta}(t)>0$, for all $t \geq 0$, then $U(t, \theta), 0 \leq \theta<t<\infty$, is a family of compact evolution operators in $x$.

Proof: (i) Define $h(t)=\int_{0}^{t} \beta(s) d s, t \geq 0$. Then for $\xi \in D(A)$, the Cauchy problem: $(d / d t) y=\beta(t) A y, t \geq 0, y(0)=\xi$, has a unique strong solution $y(t)=T(h(t)) \xi, t \geq 0$. Hence, by density of $D(A)$, the problem has a mild solution for each $\xi \in X$ given by the same expression. Then the evolution equation (2.1) can be written as a Volterra integral equation

$$
x(t)=T(h(t)) x_{0}+\int_{0}^{t} T(h(t)-h(s)) C(s) x(s) d s, \quad t \in I_{T} \equiv[0, T], \quad T<\infty .
$$

Introduce the operator $G$ with values

$$
(G z)(t) \equiv T(h(t)) x_{0}+\int_{0}^{t} T(h(t)-h(s)) C(s) z(s) d s, \quad t \in I_{T} .
$$

By virtue of strong continuity of the semigroup $T$ and continuity of $h$ and the fact that $C \in$ $L_{1}\left(I_{T}, \mathcal{L}(X)\right)$, it follows that $G$ is a continuous linear operator in $C\left(I_{T}, X\right)$. For $x, y \in C\left(I_{T}, X\right)$, define

$$
\rho_{T}(x, y) \equiv \operatorname{Sup}\left\{\exp (-\omega h(t))\|x(t)-y(t)\|_{X}, t \in I_{T}\right\}
$$

This defines a consistent metric on the Banach space $C\left(I_{T}, X\right)$. Define

$$
c(t) \equiv \int_{0}^{t}\|C(s)\|_{\mathcal{L}(X)} d s, \quad t \geq 0 .
$$

Using repeated substitution it is easy to verify that

$$
\rho_{T}\left(G^{n} x, G^{n} y\right) \leq\left((M c(T))^{n} /(n-1) !\right) \rho_{T}(x, y)
$$

for every pair $x, y \in C\left(I_{T}, X\right)$ and every positive integer $n$. Hence for sufficiently large $n, G^{n}$ is a contraction in $C\left(I_{T}, X\right)$ and by Banach fixed point theorem, $G$ has a unique fixed point in $C\left(I_{T}, X\right)$ which is the unique solution of the integral equation (2.2). Thus the Cauchy problem (2.1) has a unique mild solution for every finite $T$. The existence and uniqueness immediately implies that there exists an evolution operator $U(t, s), 0 \leq s<t<\infty$ in $X$ so that for each $\xi \in X$ it satisfies the equation

$$
U(t, s) \xi=T(h(t)-h(s)) \xi+\int_{s}^{t} T(h(t)-h(r)) C(r) U(r, s) \xi d r
$$

for all $0 \leq s<t<\infty$, satisfying

(1) $\quad U(t, t)=I$,

(2) $\quad U(t, s) U(s, \tau)=U(t, \tau), 0 \leq \tau \leq s \leq t<\infty$ 
and

(3) for $\xi \in D(A),(\partial / \partial t) U(t, s) \xi=\mathcal{A}(t) U(t, s) \xi$ for $0 \leq s<t<\infty$.

Further, for $0 \leq s \leq t<\infty$,

$$
\|U(t, s)\|_{\mathcal{L}(X)} \leq M \exp \left(\int_{s}^{t}\left[M\|C(\theta)\|_{\mathcal{L}(X)}+\omega \beta(\theta)\right] d \theta\right) .
$$

Note that, in case $C$ is constant and $\beta(t) \equiv 1$, this result coincides with the well known result for bounded perturbations of $C_{0}$-semigroups [1].

Now we prove the second part. (ii) Since $T(t), t>0$, is compact and $\beta(t)>0$ for all $t \geq 0$ and it is locally integrable, we conclude that $T(h(t)-h(s)), 0 \leq s<t<\infty$, is a family of compact operators in $X$. Define $U_{\epsilon}(t, s)$ and $R_{\epsilon}(t, s)$ by

$$
\begin{gathered}
U_{\epsilon}(t, s) \equiv T(h(t)-h(s))+\int_{s}^{t-\epsilon} T(h(t)-h(r)) C(r) U(r, s) d r \\
R_{\epsilon}(t, s) \equiv \int_{t-\epsilon}^{t} T(h(t)-h(r)) C(r) U(r, s) d r
\end{gathered}
$$

for $0 \leq s<t-\epsilon \leq t<\infty$. By (2.4) $U$ is bounded and since, by our assumption (ii), $C(t)$ is a family of (essentially) bounded operators, $U_{\epsilon}(t, s), 0 \leq s<t<\infty$ is a family of compact operators in $X$. Further it is easy to verify that the family of operators $R_{\epsilon}(t, s)$ converges to 0 in the uniform operator topology. Thus $U_{\epsilon}(t, s) \rightarrow U(t, s)$ in the uniform operator topology proving that $U(t, s), 0 \leq s<t<\infty$ is a family of compact evolution operators in $X$. This completes the proof.

In the following corollary we extend this result to semilinear problems with Lipschitz nonlinearities.

Corollary 2.2: $\quad$ Consider the system

$$
\begin{gathered}
(d / d t) x=\beta(t) A x+C(t) x+f(x), t \geq s \\
x(s)=\zeta, \quad 0 \leq s<\infty
\end{gathered}
$$

where $A$ and $\beta$ satisfy the assumptions of Theorem 2.1 and $f$, a mapping from $X$ to $X$, is locally Lipschitzian and satisfies a global linear growth condition. Then,

(i) under the assumptions of Theorem 2.1(i), there exists a nonlinear strongly continuous (Lipschitzian on $X$ ) evolution operator $N(t, \tau), 0 \leq \tau \leq t<\infty$ in $X$ which solves the Cauchy problem (2.6) in the mild sense, that is $x(t)=N(t, s) \zeta$, $t \geq s$;

(ii) under the assumptions of Theorem 2.1(ii), the nonlinear evolution operator $N(t, \tau)$, $0 \leq \tau<t<\infty$ is compact.

Proof: By virtue of Theorem 2.1, the mild solution for the Cauchy problem (2.6) is given by the solution of the integral equation

$$
x(t)=U(t, s) \zeta+\int_{s}^{t} U(t, r) f(x(r)) d r
$$

where $U$ is the evolution operator corresponding to the operator $\mathcal{A}(t) \equiv \beta(t) A+C(t)$ as given by Theorem 2.1. Under the global linear growth assumption, it is easy to verify that a solution, if any exists, is norm bounded in $C\left(I_{T}, X\right)$. Using this bound and the local Lipschitz property one 
can conclude through Banach fixed point theorem, as in Theorem 2.1, that this equation has a unique solution $x(t, s, \zeta), \infty \geq t \geq s \geq 0$, which is strongly continuous in $t$ for $t \geq s$ and Lipschitzian in $\zeta$. Define $N(t, s) \zeta \equiv x(t, s, \zeta)$ for $t \geq s \geq 0$. This is a nonlinear evolution operator satisfying

(a) $\quad N(t, t)=I$,

(b) $\quad N(t, s)=N(t, r) N(r, s), 0 \leq s \leq r \leq t$,

(c) $s-\lim _{t \downarrow s} N(t, s) \zeta=\zeta$.

This proves the first part. For the second part, note that $U$ is a compact linear evolution and hence the linear operator $L_{t}$ given by $\left(L_{t} y\right) \equiv \int_{s}^{t} U(t, r) y(r) d r, 0 \leq s \leq t \leq T$, defines a compact map from $C([s, t], X)$ to $X$. Since $f$ satisfies the linear growth condition and is locally Lipschitzian, the Nemytski operator $F$, given by $F x(\cdot) \equiv f(x(\cdot))$, is a continuous bounded map in the Banach space $C(I, X)$ for any finite interval $I \subset[0, \infty)$. Hence $L_{t} F$ is a compact operator from $C([s, t], X)$ to $X$. Thus $N(t, s), 0 \leq s<t<\infty$ is a strongly continuous compact nonlinear evolution in $X$. This completes the proof.

In the following theorem we present a result on the dependence of solutions with respect to the perturbation $\beta \in L_{1}^{l o c}(0, \infty)$.

Theorem 2.3: $\quad$ Consider the system

$$
\begin{gathered}
(d / d t) x=\beta(t) A x+f(x), \quad t \geq 0 \\
x(0)=\zeta, \quad 0 \leq t<\infty .
\end{gathered}
$$

Suppose $f$ satisfies the assumption of Corollary 2.2 and $A \in G(X, M, \omega)$ with $T$ being the corresponding semigroup and $\zeta \in X$. Let $\beta(t) \geq 0, \beta \in L_{1}^{\text {loc }}(0, \infty)$ and $\left\{\beta_{n}\right\}$ be a sequence of nonnegative measurable functions belonging to $L_{1}^{\text {loc }}(0, \infty)$. Then,

(i) If $\beta_{n} \rightarrow \beta$ weakly, the solution $x_{n}$ of the Cauchy problem (2.8) corresponding to $\beta=\beta_{n}$ converges pointwise (not uniformly) in $t$ on bounded intervals to the mild solution $x$ of $(2.8)$.

(ii) If $\beta_{n} \rightarrow \beta$ strongly in $L_{1}(I)$ on any finite interval $I$ and the sequence $\left\{\beta_{n}\right\}$ is dominated by an integrable function, then $x_{n}$ converges to $x$ uniformly on $I$.

Proof: (i) Since $\beta_{n} \stackrel{w}{\rightarrow} \beta$ in $L_{1}^{\text {loc }}$, it is clear that for any finite interval $I=I_{\tau}=[0, \tau]$, $\sup \int_{I}\left|\beta_{n}\right| d t \leq b$ for some $b=b_{\tau}<\infty$ and for each $t \in I_{\tau}$,

$$
h_{n}(t) \equiv \int_{0}^{t} \beta_{n}(s) d s \rightarrow \int_{0}^{t} \beta(s) d s \equiv h(t), \quad t \in I_{\tau}, \quad \tau<\infty
$$

Let $\left\{x_{n}\right\}$ and $x$ be the mild solutions of (2.8) corresponding to $\left\{\beta_{n}\right\}$ and $\beta$ respectively. Since $f$ satisfies the global linear growth condition, there exists an $R<\infty$ and a ball $B_{R} \subset X$ of radius $R$, centered at the origin, such that $x_{n}(t), x(t) \in B_{R}$ for all $t \in I$ and $n \in N \equiv\{1,2,3, \ldots\}$. Write

$$
x(t)-x_{n}(t) \equiv z_{1}^{n}(t)+z_{2}^{n}(t)+z_{3}^{n}(t),
$$

where

$$
\begin{gathered}
z_{1}^{n}(t) \equiv T(h(t)) \zeta-T\left(h_{n}(t)\right) \zeta \\
z_{2}^{n}(t) \equiv \int_{0}^{t}\left(T(h(t-s))-T\left(h_{n}(t-s)\right)\right) f(x(s)) d s
\end{gathered}
$$




$$
z_{3}^{n}(t) \equiv \int_{0}^{t} T\left(h_{n}(t-s)\right)\left(f(x(s))-f\left(x_{n}(s)\right)\right) d s
$$

Since $T$ is a $C_{0}$-semigroup, $z_{1}^{n}(t) \rightarrow 0$ as $n \rightarrow \infty$ for each $t \in I$. The integrand in the expression for $z_{2}^{n}(t)$ converges to zero pointwise, and it is dominated by an integrable function, since, due to the weak convergence of $\beta_{n}, S u p_{n}\left|h_{n}(t)\right|<\infty$ for each $t \in I$, and $x(t) \in B_{R}$ for $t \in I$, and $T$ is bounded on bounded intervals. Hence by Lebesgue dominated convergence theorem, $z_{2}^{n}(t) \rightarrow 0$, for each $t \in I$. By using the local Lipschitz property of $f$, and the fact that $x_{n}(t)$ and $x(t)$ belong to $\in B_{R}$ for all $t \in I$, as mentioned earlier, we have

$$
\left\|x(t)-x_{n}(t)\right\| \leq \alpha_{n}(t)+C \int_{0}^{t}\left\|x(s)-x_{n}(s)\right\| d s
$$

where $\alpha_{n}(t)=\left\|z_{1}^{n}(t)\right\|+\left\|z_{2}^{n}(t)\right\|$, and $C$ is a constant dependent only on $b, R, \omega, M$ and $\tau$. Since $\alpha_{n}(t) \rightarrow 0$ for each $t \in I$ and is uniformly bounded on $I$, it follows from the generalized Gronwall inequality that $x_{n}(t) \rightarrow x(t)$ pointwise for each $t \in I$. Since $I=I_{\tau}, \tau<\infty$, we conclude that the convergence holds pointwise for each $t \in[0, \infty)$.

(ii) For the second part, note that, for any $\xi \in D(A)$,

$$
\begin{gathered}
T\left(h_{n}(t)\right) \xi-\xi=\int_{0}^{t} \beta_{n}(s) T\left(h_{n}(s)\right) A \xi d s \\
T(h(t)) \xi-\xi=\int_{0}^{t} \beta(s) T(h(s)) A \xi d s
\end{gathered}
$$

for $t \in I_{\tau}, \tau<\infty$. Thus for $\xi \in D(A)$, and $\tau<\infty$, we have

$$
\begin{aligned}
S u p_{0} \leq t \leq \tau & T\left(h_{n}(t)\right) \xi-T(h(t)) \xi\left\|\leq \int_{0}^{\tau}\left|\beta(s)-\beta_{n}(s)\right|\right\| T(h(s)) A \xi \| d s \\
& +\int_{0}^{\tau}\left|\beta_{n}(s)\right|\left\|\left(T(h(s))-T\left(h_{n}(s)\right)\right) A \xi\right\| d s .
\end{aligned}
$$

Since $\beta_{n} \stackrel{s}{\rightarrow} \beta$ and $\beta_{n}$ is dominated by an integrable function, using the dominated convergence theorem once again it follows from the above inequality that

$$
T\left(h_{n}(t)\right) \xi \rightarrow T(h(t)) \xi \text { uniformly in } t \in I_{\tau}, \quad \tau<\infty
$$

for each $\xi \in D(A)$ and, hence, by virtue of density of $D(A)$ in $X$, this holds for each $\xi \in X$. As a result, in this case $\alpha_{n}(t) \rightarrow 0$ uniformly on $I_{\tau}$. Then, using Gronwall inequality once again, we obtain

$$
S u p_{t \in I_{\tau}}\left\|x(t)-x_{n}(t)\right\| \leq \exp (C \tau) S u p_{t \in I_{\tau}}\left(\alpha_{n}(t)\right) .
$$

It follows from this estimate that $x_{n}(t) \rightarrow x(t)$ uniformly in $t \in I_{\tau}$ for each finite $\tau$. This completes the proof.

Remark: In case the operator $A$ of equation (1.1) is the infinitesimal generator of a group 
of linear operators, $T(t), t \in R$, in $X$, the multiplicative perturbation $\beta$, can be any locally integrable function. In other words, positivity assumption is no longer necessary.

\section{A System of Evolutions and Periodic Solutions}

Now we consider the system of evolution equations of the form

$$
\begin{aligned}
& (d / d t) x=\beta(t) A x+C_{11}(t) x+C_{12}(t) y, x(0)=x_{0} \\
& (d / d t) y=\gamma(t) B y+C_{21}(t) x+C_{22}(t) y, \quad y(0)=y_{0}
\end{aligned}
$$

in a pair of Banach spaces $X$ and $Y$.

Theorem 3.1: Let $A \in G\left(X, M_{1}, \omega_{1}\right)$ and $B \in G\left(Y, M_{2}, \omega_{2}\right)$ with the corresponding semigroups denoted by $T$ and $S$ respectively. Suppose $C_{11} \in L_{1}^{\text {loc }}([0, \infty), \mathcal{L}(X)), C_{12} \in$ $L_{1}^{\text {loc }}([0, \infty), \mathcal{L}(Y, X)), \quad C_{21} \in L_{1}^{\text {loc }}([0, \infty), \mathcal{L}(X, Y)) \quad$ and $C_{22} \in L_{1}^{\text {loc }}([0, \infty), \mathcal{L}(Y))$ and let $\beta$, $\gamma \in L_{1}^{\text {loc }}([0, \infty))$ and nonnegative. Then for any $x_{0} \in X$ and $y_{0} \in Y$, the following holds true:

(i) if $\beta$ and $\gamma$ are nonnegative, the system (3.1) has a unique solution in $C(I, X \times Y)$ for any finite interval $I \equiv[0, T]$ and defines a strongly continuous linear evolution operator $V(t, \tau), 0 \leq \tau \leq t<\infty$, in $X \times Y$;

(ii) if both $\beta, \gamma$ are strictly positive and the semigroups $T$ and $S$ are compact, the corresponding evolution operator $V(t, \tau), 0 \leq \tau<t<\infty$, is also compact.

Proof: Introduce the Banach space $Z \equiv X \times Y$, with the norms given by

$$
\|z\|_{Z} \equiv\|x\|_{X}+\|y\|_{Y}
$$

Defining

and

$$
\mathcal{A}_{0}(t) \equiv\left(\begin{array}{cc}
\beta(t) A & 0 \\
0 & \gamma(t) B
\end{array}\right)
$$

$$
\mathcal{e}(t) \equiv\left(\begin{array}{ll}
C_{11}(t) & C_{12}(t) \\
C_{21}(t) & C_{22}(t)
\end{array}\right)
$$

one can rewrite system (3.1) as one equation in the Banach space $Z$ as follows:

$$
\begin{gathered}
(d / d t) z=\mathcal{A}_{0}(t) z+\mathcal{C}(t) z, \\
z(0) \equiv z_{0} \equiv\left(\begin{array}{c}
x_{0} \\
y_{0}
\end{array}\right) .
\end{gathered}
$$

Under the given assumptions, $\mathcal{A}_{0}$ generates a strongly continuous evolution operator in $Z$ and $e \in L_{1}^{l o c}([0, \infty), \mathcal{L}(Z))$. The conclusions of the theorem then follow Theorem 2.1. This completes the proof.

Adding a nonlinear coupling term like

$$
F(z)=\left(\begin{array}{l}
f_{1}(x, y) \\
f_{2}(x, y)
\end{array}\right)
$$


in the evolution system (3.2) we can prove the following result.

Corollary 3.2: $\quad$ Consider the system (3.4):

$$
\begin{gathered}
(d / d t) z=\mathcal{A}_{0}(t) z+\mathrm{C}(t) z+F(z), \quad t \geq s \geq 0 ; \\
z(s)=\zeta .
\end{gathered}
$$

Suppose the assumptions of Theorem 3.1 hold and the nonlinear maps $f_{1}: Z \rightarrow X$ and $f_{2}: Z \rightarrow Y$ are locally Lipschitzian satisfying the global linear growth condition. Then,

(i) under the assumptions of Theorem 3.1(i), there exists a nonlinear strongly continuous (Lipschitzian on $Z$ ) evolution operator $N(t, s), 0 \leq s \leq t<\infty$ in $Z$ which solves the Cauchy problem (3.4) in the mild sense, that is $z(t)=N(t, s) \zeta, t \geq s$;

(ii) under the assumptions of Theorem 3.1(ii), the nonlinear evolution operator $N(t, s)$, $0 \leq s<t<\infty$, is compact.

Proof: The proof is identical to that of Corollary 2.2.

Next we present two results on the existence of periodic solutions of the nonlinear evolution system. This is important in many Physical and Biological sciences. Co-existence of various cooperating and competing species in the nature and their population fluctuation indicate periodicity in the dynamics. Also in the climatology the periodicity is a common phenomenon.

Theorem 3.3: $\quad$ Consider the system;

$$
(d / d t) z=\mathcal{A}_{0}(t) z+\mathrm{C}(t) z+F(z), \quad t \geq 0 ;
$$

and suppose the following assumptions hold:

$(A 1): A \in G(X, M,-\omega)$ and $B \in G(Y, M,-\omega)$, for some $\omega>0$;

(A2) $\beta$ and $\gamma$ and the operators $\left\{C_{i, j}, i, j=1,2\right\}$ satisfy the assumptions of Theorem 3.1, and further they are all $\tau$-periodic $(\tau>0)$;

(A3) there exist constants $a, b>0$, such that

$$
\begin{gathered}
\|F(z)\|_{Z} \leq a+b\|z\|_{Z} \text { for all } z \in Z \\
\left\|F(z)-F\left(z^{*}\right)\right\|_{Z} \leq b\left\|z-z^{*}\right\|_{Z} \text {, for all } z, z^{*} \in Z ;
\end{gathered}
$$

(A4) there exists a $\delta>0$ such that

$$
\ell_{n}(M)+\int_{0}^{\tau}\left\{M\left[b+\|\mathcal{C}(t)\|_{\mathcal{L}(Z)}\right]-\omega \eta(t)\right\} d t=-\delta
$$

where $\eta(t) \equiv \beta(t) \wedge \gamma(t)$ and $\ell_{n}$ stands for the natural logarithm.

Then there exists an $R_{0}>0$ such that system (3.5) has a unique $\tau$-periodic solution $\tilde{z}(t), t \geq 0$, that resides in the ball $B_{R_{0}}$ of $Z$ centered at the origin, and further, this solution is globally exponentially stable.

Proof: By virtue of Theorem 3.1, $\mathcal{A}_{0}(t)+\mathrm{C}(t)$ generates an evolution operator $V(t, s), 0 \leq$ $s \leq t<\infty$, in the Banach space $Z$. Under assumptions $(A 1)-(A 2)$, it is easy to verify that

$$
\|V(t, s)\|_{\mathcal{L}(Z)} \leq M \exp \int_{s}^{t}\left(M\|\mathrm{C}(r)\|_{\mathcal{L}(Z)}-\omega \eta(r)\right) d r
$$


for all $0 \leq s \leq t<\infty$. For convenience, define

$$
g(s) \equiv(\omega \eta(s)-M\|\mathrm{C}(s)\|), s \geq 0 .
$$

Using assumption ( $A 3$ ), it follows from Corollary 3.2 that, for each $\zeta \in Z$, the integral equation

$$
z(t)=V(t, 0) \zeta+\int_{0}^{t} V(t, r) F(z(r)) d r
$$

has a unique solution $z(t) \equiv z(t, \zeta), t \geq 0, z \in C([0, \tau], Z)$. Then, using Gronwall's inequality, one can verify that

for all $t \in[0, \tau]$. Define

$$
\|z(t, \zeta)\| \leq M \exp \left\{\int_{0}^{t}[M b-g(r)] d r\right\}\left(\|\zeta\|_{Z}+a\left(\int_{0}^{\tau}\left(\exp \int_{0}^{r} g(s) d s\right) d r\right),\right.
$$

$$
\begin{gathered}
R_{0} \equiv M d e /(1-M d), \text { where } \\
d \equiv \exp \left(\int_{0}^{\tau}[M(b+\|\mathrm{C}(s)\|)-\omega \eta(s)] d s\right) \\
e \equiv a \int_{0}^{\tau}\left(\exp \int_{0}^{s} g(r) d r\right) d s .
\end{gathered}
$$

By virtue of assumption $(A 3)$ and (3.6), $0<M d<1$, and hence, $R_{0}>0$. Consider the ball $B_{R_{0}} \subset Z$ of radius $R_{0}$ centered at the origin. It follows from (3.8) that, the nonlinear map $\Gamma_{\tau}$, given by

$$
\Gamma_{\tau} \xi \equiv z(\tau, \xi)=N(\tau, 0) \xi
$$

maps $B_{R_{0}}$ into itself. Further, by using the Lipschitz property of $F$, and the Gronwall inequality, one can verify that

$$
\left.\| \Gamma_{\tau} \xi_{1}-\Gamma_{\tau} \xi_{2}\right)\left\|_{Z} \leq M d\right\| \xi_{1}-\xi_{2} \|_{Z}
$$

Since $M d<1, \Gamma_{\tau}$ is a contraction and hence by Banach fixed point theorem, it has a nontrivial fixed point $\zeta_{0} \in B_{R_{0}}$. Therefore, the function given by $\widetilde{z}(t) \equiv N(t, 0) \zeta_{0}, t \geq 0$, is a periodic solution of the evolution system (3.5). It remains to prove the stability of the solution. Suppose the periodic solution $\widetilde{z}$ is perturbed at time $t_{0} \geq 0$ and let $z$ be the perturbed trajectory. Then the trajectory $y$, given by $y \equiv z-\widetilde{z}$, satisfies the evolution equation

$$
\begin{gathered}
(d / d t) y=\mathcal{A}_{0}(t) y+\mathcal{C}(t) y+\tilde{F}(t, y), t \geq t_{0} \\
y_{0}=\gamma \neq 0,
\end{gathered}
$$

where $\tilde{F}(t, \xi) \equiv F(\tilde{z}(t)+\xi)-F(\tilde{z}(t))$. Then by virtue of estimate (3.7) and inequality (3.6), it follows that

$$
\|y(t)\| \leq M\|\gamma\| \exp -\delta\left(t-t_{0}\right), \text { for all } t \geq t_{0} .
$$

This proves the exponential stability of the periodic solution and thereby completes the proof of the theorem. 
Remark: Theorem 3.3 also holds for $F=F(t, z), t \geq 0, z \in Z$, provided it is $\tau$-periodic in $t$ and assumption (A3) holds uniformly with respect to $t \geq 0$.

The result of Theorem 3.3 can be extended to a locally Lipschitzian $F$ that satisfies linear growth condition. This is given in the following theorem.

Theorem 3.4: $\quad$ Consider system (3.5) and suppose the following assumptions hold:

(a1) $X$ and $Y$ are uniformly convex and $Z \equiv X \times Y$ is given the topology which is induced by any of the following norms,

$$
\|z\|_{Z} \equiv\left(\|x\|_{X}^{p}+\|y\| \stackrel{p}{Y}\right)^{1 / p}, 2 \leq p<\infty, x \in X \text { and } y \in Y
$$

(a2) $A \in G(X, M,-\omega), B \in G(Y, M,-\omega)$ for some $\omega>0,0 \leq \beta, \gamma \in L_{1}^{\text {loc }}([0, \infty))$ and $\tau$-periodic,

(a3) $\quad C_{11} \in L_{p}^{l o c}([0, \infty), \mathcal{L}(X)), C_{22} \in L_{p}^{l o c}([0, \infty), \mathcal{L}(Y)), C_{12} \in L_{p}^{l o c}([0, \infty), \mathcal{L}(Y, X))$ and $C_{21} \in L_{p}^{\text {Poc }}([0, \infty), \mathcal{L}(X, Y))$, and they are $\tau$-periodic,

(a4) $F$ has atmost the linear growth: $\|F(z)\| \leq a+b\|z\|, a>0, b>0$, with the growth rate satisfying:

$$
\ell_{n}(M)+\int_{0}^{\tau}\left\{M\left[b+\|\mathcal{C}(t)\|_{\mathcal{L}(Z)}\right]-\omega \eta(t)\right\} d t \leq 0 ;
$$

(a5) $F$ is locally Lipschitzian and there exists an $R \geq R_{0} \equiv M d e /(1-M d)$ such that

$$
\mathcal{L}_{n}(M)+\int_{0}^{\tau}\left\{M\left[K_{R}+\|\mathcal{C}(t)\|_{\mathcal{L}(Z)}\right]-\omega \eta(t)\right\} d t \leq 0 ;
$$

where $K_{R} \equiv \operatorname{Sup}\left\{\|F(x)-F(y)\| /(\|x-y\|), x \neq y, x, y \in B_{R}\right\}$, with $B_{R}$ being the ball of radius $R$ in $Z$ centered at the origin.

Then system (3.5) has at least one $\tau$-periodic solution $\widetilde{z}$ such that $\widetilde{z}(t) \in B_{R}$ for all $t \geq 0$.

Proof: We outline the proof indicating the main differences with that of Theorem 3.3. Under the assumption (a1)-(a4), one can verify as in Theorem 3.3, that the nonlinear map $\Gamma_{\tau}$ maps $B_{R}$ into itself for each $R \geq R_{0}$. Under assumption (a5), $\Gamma_{\tau}$ is a nonexpansive map in $B_{R}$. Since $Z$ is uniformly convex with respect to any of the given norm topologies, it follows from the fixed point theorem for nonexpansive maps due to Browder [2], that $\Gamma_{\tau}$ has at least one fixed point in the ball $B_{R} \subset Z$. Hence system (3.5) has at least one $\tau$-periodic solution. In fact, the result is also true for reflexive Banach spaces having normal structure. This is due to a fixed point theorem of Kirk [4, Theorem 4.1]. This completes the proof.

\section{References}

[1] Ahmed, N.U., Semigroup Theory with Applications to Systems and Control, Pitman Res. Notes in Math. Ser. 246, Longman Scientific and Technical and John Wiley, London, New York 1991.

[2] Browder, F.E., Nonexpansive nonlinear operators in Banach spaces, Proc. Nat. Acad. Sci. USA 54 (1965), 1041-1044.

[3] Friedman, A., Stochastic Differential Equations and Applications, Vol. 1, 2, Academic Press, New York, San Francisco, London 1975.

[4] Goebel, K., and Kirk, W.A., Topics in Metric Fixed Point Theory, Cambridge Studies in Advanced Mathematics, vol. 281991. 
[5] Goldstein, J.A., Semigroups of Linear Operators and Applications, Oxford University Press, Oxford, New York 1985.

[6] Ladas, G.E., and Lakshmikantham, V., Differential Equations in Abstract Spaces, Math. in Science and Engineering, Vol. 85, Academic Press, New York and London 1972. 


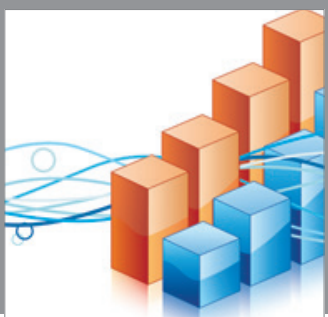

Advances in

Operations Research

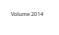

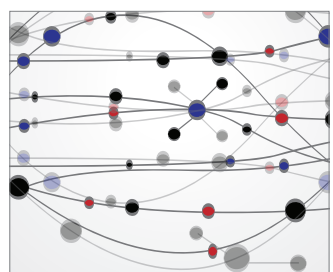

\section{The Scientific} World Journal
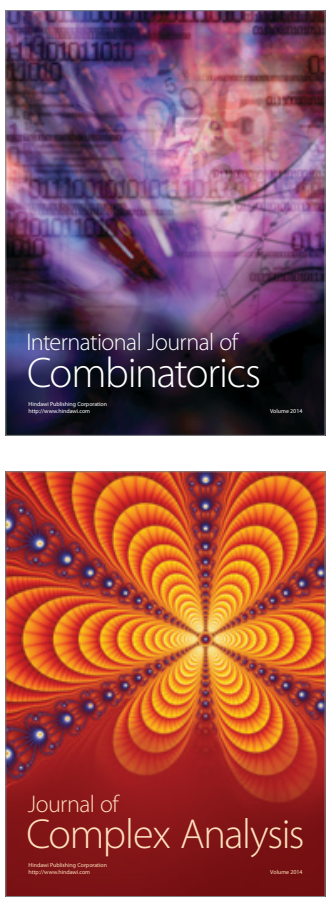

International Journal of

Mathematics and

Mathematical

Sciences
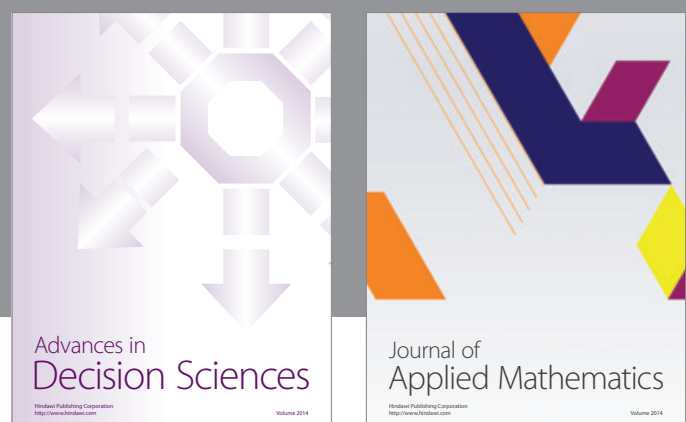

Journal of

Applied Mathematics
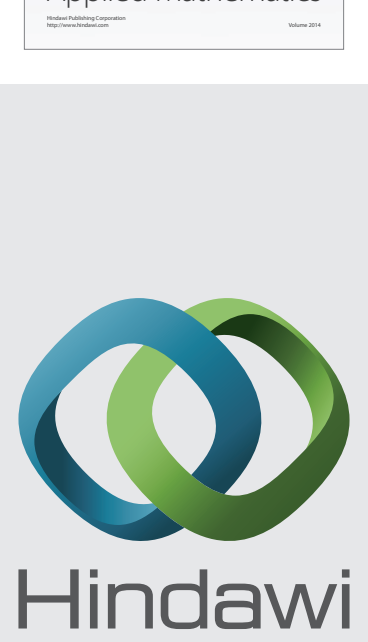

Submit your manuscripts at http://www.hindawi.com
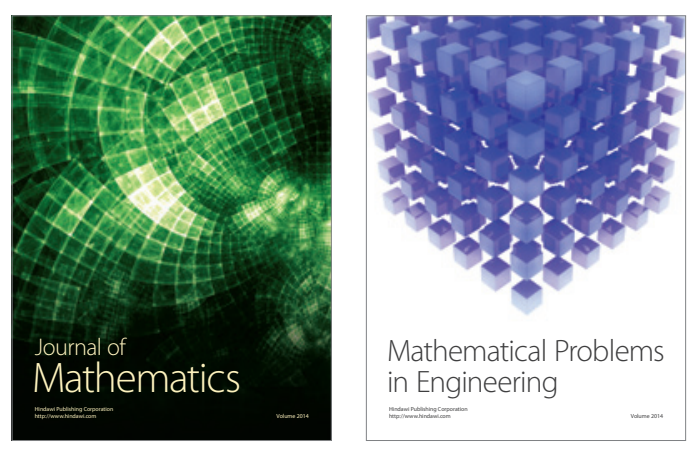

Mathematical Problems in Engineering
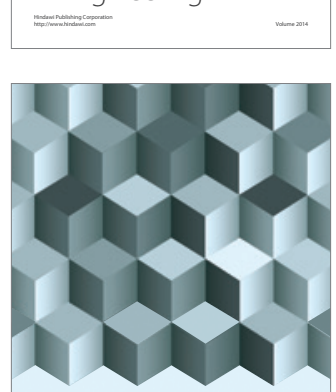

Journal of

Function Spaces
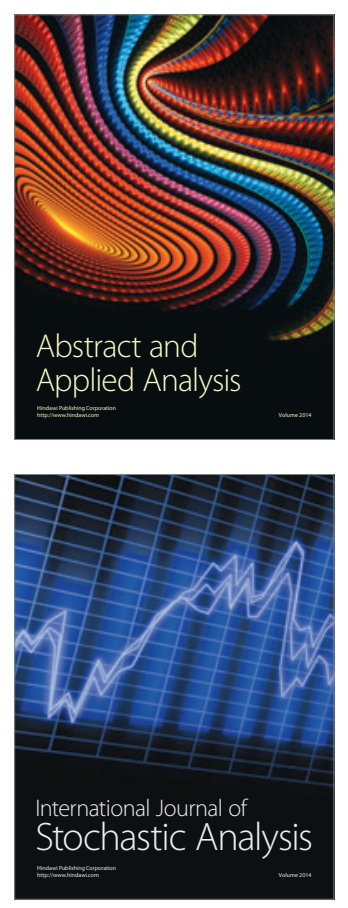

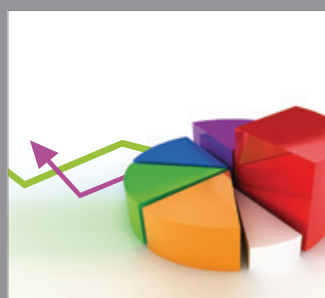

ournal of

Probability and Statistics

Promensencen
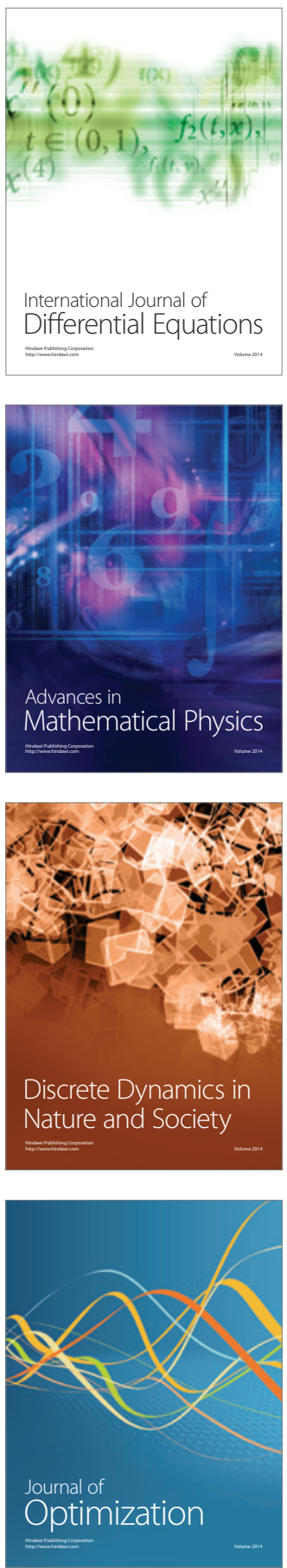